\title{
Defining and investigating new soft ordered maps by using soft semi open sets
}

\author{
Tareq M. Al-shami \\ Department of Mathematics, Sana'a University, \\ Sana'a- Yemen \\ email: tareqalshami83@gmail.com
}

\begin{abstract}
Here, we employ soft semi open sets to define new soft ordered maps, namely soft $x$-semi continuous, soft $x$-semi open, soft $x$-semi closed and soft $x$-semi homeomorphism maps, where $x$ denotes the type of monotonicity. To show the relationships among them, we provide some illustrative examples. Then we give complete descriptions for each one of them. Also, we investigate "transmission" of these maps between soft and classical topological ordered spaces.
\end{abstract}

\section{Introduction}

In 1965, Nachbin [41] introduced new mathematical structure, namely topological ordered space. This structure consists of two independent concepts defined on a non-empty set $X$, one of them is a topological space $(X, \tau)$ and the other is a partially ordered set $(X, \preceq)$. McCartan [39] in 1968, studied separation axioms via topological ordered spaces. Kumar [35] defined the concepts of continuous and homeomorphism maps via topological ordered spaces. Recently, the authors of $[1,4,6,9,10,12,23,26,28]$ have introduced and investigated many concepts via supra topological ordered spaces.

In 1999, Molotdsov [40] introduced the concept of soft sets for dealing with uncertainties and vagueness. Then, Maji et al. [38] put up the basis of soft

2010 Mathematics Subject Classification: 54F05, 54F15

Key words and phrases: soft I(D, B)-semi continuous map, soft I(D, B)-semi open map, soft I(D, B)-semi closed map, soft I(D, B)-semi homeomorphism map 
set theory by defining some operations between two soft sets like soft subset and equality relations, and soft union and intersection. Shabir and Naz [45] initiated the idea of soft topological spaces and studied soft separation axioms. Later on, many researchers carried out several studies to discuss the topological notions on soft topologies (see, for example [2, 3, 7, 11, 15, 20, 22, 29, 30, 31, 36, 44]). Chen [24] and Mahanta and Das[37] displayed and probed the notions of soft semi open sets and soft semi separation axioms. Depend on soft semi open sets, some works were done (see, for example [5, 25, 33, 34]). At present, the notions of soft topological ordered spaces [16], supra soft topological ordered spaces [13] and soft ordered maps [13] were introduced and investigated.

This paper is organized as follows: In Section (2), we recall the previous definitions and results that we will need to prove our results. Section (3) gives other applications of soft semi open sets by defining some soft ordered maps, namely soft $x$-semi continuous, soft $x$-semi open, soft $x$-semi closed and soft $\chi$-semi homeomorphism maps for $x \in\{I, D, B\}$. These concepts are described and some examples are constructed to show the relationships among them. Also, we demonstrate the interrelationships between these soft maps and their counterparts of crisp ordered maps when the soft topology is extended. Section (4) concludes the paper.

\section{Preliminaries}

Let $X$ and $\Omega$ be a universal set and a set of parameters, respectively. The power set of $X$ is denoted by $2^{X}$.

\subsection{Soft sets}

Definition 1 [40] A notation $\mathrm{G}_{\Omega}$ is said to be a soft set, terminologically, over $\mathrm{X}$, if $\mathrm{G}$ is a map from $\Omega$ to $2^{\mathrm{X}}$. Usually, we write it as follows:

$$
\mathrm{G}_{\Omega}=\left\{(\omega, \mathrm{G}(\omega)): \omega \in \Omega \text { and } \mathrm{G}(\omega) \in 2^{\mathrm{X}}\right\} .
$$

Through this article, $\mathrm{S}\left(\mathrm{X}_{\Omega}\right)$ denotes the family of soft sets on $\mathrm{X}$ with $\Omega$.

Definition $2[29,45]$ For $\mathrm{y} \in \mathrm{X}$ and $\mathrm{G}_{\Omega}$ over $\mathrm{X}$, we write that:

1. $\mathrm{y} \in \mathrm{G}_{\Omega}$ (resp. $\left.\mathrm{y} \notin \mathrm{G}_{\Omega}\right)$ if $\mathrm{y} \in \mathrm{G}(\omega)$ (resp. $\mathrm{y} \notin \mathrm{G}(\omega)$ ) for each $\omega \in \Omega$.

2. $\mathrm{y} \Subset \mathrm{G}_{\Omega}$ (resp. $\left.\mathrm{y} \notin \mathrm{G}_{\Omega}\right)$ if $\mathrm{y} \in \mathrm{G}(\omega)$ (resp. $\mathrm{y} \notin \mathrm{G}(\omega)$ ) for some $\omega \in \Omega$. 
Definition 3 [38] If $\mathrm{G}(\omega)=\emptyset$ and $\mathrm{F}(\omega)=X$ for each $\omega \in \Omega$, then $\mathrm{G}_{\Omega}$ and $\mathrm{F}_{\Omega}$ are respectively called null soft set and absolute soft set. They are respectively denoted by $\widetilde{\emptyset}$ and $\widetilde{\mathrm{X}}$.

Definition 4 [21] The relative complement $\mathrm{G}_{\Omega}^{\mathrm{c}}$ of $\mathrm{G}_{\Omega}$ is defined by $\mathrm{G}^{\mathrm{c}}(\omega)=$ $\mathrm{X} \backslash \mathrm{G}(\omega)$ for each $\omega \in \Omega$.

Definition 5 [46] A soft mapping of $\mathrm{S}\left(\mathrm{X}_{\Omega},\right)$ into $\mathrm{S}\left(\mathrm{Y}_{\Gamma}\right)$, denoted by $\mathrm{f}_{\phi}$, is a pair of mappings $\mathrm{f}: \mathrm{X} \rightarrow \mathrm{Y}$ and $\phi: \Omega \rightarrow \Gamma$ such that the image of $\mathrm{G}_{\mathrm{K}} \in \mathrm{S}\left(\mathrm{X}_{\Omega},\right)$ and pre-image of $\mathrm{H}_{\mathrm{L}} \in \mathrm{S}\left(\mathrm{Y}_{\Gamma}\right)$ are given by the following formulations:

(i) $\mathrm{f}_{\phi}\left(\mathrm{G}_{\mathrm{K}}\right)=\left(\mathrm{f}_{\phi}(\mathrm{G})\right)_{\Gamma}$ is a soft subset of $\mathrm{S}\left(\mathrm{Y}_{\Gamma}\right)$ given by

$$
f_{\phi}(G)(\gamma)=\left\{\begin{array}{ccc}
\bigcup_{a \in \phi^{-1}(\gamma) \cap K} f(G(a)) & : & \phi^{-1}(\gamma) \bigcap K \neq \emptyset \\
\emptyset & : & \phi^{-1}(\gamma) \bigcap K=\emptyset
\end{array}\right.
$$

for each $\gamma \in \Gamma$.

(ii) $\mathrm{f}_{\phi}^{-1}\left(\mathrm{H}_{\mathrm{L}}\right)=\left(\mathrm{f}_{\phi}^{-1}(\mathrm{H})\right)_{\Omega}$ is a soft subset of $\mathrm{S}\left(\mathrm{X}_{\Omega}\right)$ given by

$$
f_{\phi}^{-1}(H)(\omega)=\left\{\begin{array}{cll}
f^{-1}(H(\phi(\omega))) & : & \phi(\omega) \in L \\
\emptyset & : & \phi(\omega) \notin L
\end{array}\right.
$$

for each $\omega \in \Omega$.

Definition 6 [46] If $\mathrm{f}$ and $\phi$ are injective (resp. surjective, bijective) maps, then $\mathrm{f}_{\phi}: \mathrm{S}\left(\mathrm{X}_{\Omega}\right) \rightarrow \mathrm{S}\left(\mathrm{Y}_{\Gamma}\right)$ is said to be injective (resp. surjective, bijective).

Proposition 1 [42] Let $\mathrm{G}_{\Omega}$ and $\mathrm{H}_{\Gamma}$ be soft subsets of $\mathrm{S}\left(\mathrm{X}_{\Omega}\right)$ and $\mathrm{S}\left(\mathrm{Y}_{\Gamma}\right)$, respectively. Then:

(i) $\mathrm{G}_{\Omega} \widetilde{\widetilde{\widetilde{f}}} \mathrm{f}_{\phi}^{-1} \mathrm{f}_{\phi}\left(\mathrm{G}_{\Omega}\right)$. If $\mathrm{f}_{\phi}$ is injective, then $\mathrm{G}_{\Omega}=\mathrm{f}_{\phi}^{-1} \mathrm{f}_{\phi}\left(\mathrm{G}_{\Omega}\right)$.

(ii) $\mathrm{f}_{\phi} \mathrm{f}_{\phi}^{-1}\left(\mathrm{H}_{\Gamma}\right) \widetilde{\widetilde{C}} \mathrm{H}_{\Gamma}$. If $\mathrm{f}_{\phi}$ is surjective, then $\mathrm{f}_{\phi} \mathrm{f}_{\phi}^{-1}\left(\mathrm{H}_{\Gamma}\right)=\mathrm{H}_{\Gamma}$.

Definition 7 [27], [42] If there exist $\omega \in \Omega$ and $x \in X$ such that $\mathrm{G}(\omega)=\{x\}$ and $\mathrm{G}(\mathrm{a})=\emptyset$ for each $\mathrm{a} \in \Omega \backslash\{\boldsymbol{\omega}\}$, then $\mathrm{G}_{\Omega}$ is called a soft point. Briefly, it is denoted by $\mathrm{P}_{\omega}^{\mathrm{x}}$.

If $x \in G(\omega)$, then $P_{\omega}^{x} \in G_{\Omega}$. 
Definition $8[16]$ A triple $(\mathrm{X}, \Omega, \preceq)$ is said to be a partially ordered soft set if $(\mathrm{X}, \preceq)$ is a partially ordered set.

$\preceq$ is called linearly ordered if any pair of elements in the set of the relation are comparable, i.e., for each $\mathrm{x}, \mathrm{y} \in \mathrm{X}$ either $\mathrm{x} \preceq \mathrm{y}$ or $\mathrm{y} \preceq \mathrm{x}$.

Remark 1 Through this paper, the notation $\triangle$ denotes a diagonal relation, i.e. $\triangle=\{(x, x): x \in X\}$.

Definition 9 [16] An increasing soft operator $i:\left(\mathrm{S}\left(\mathrm{X}_{\Omega}\right), \preceq\right) \rightarrow\left(\mathrm{S}\left(\mathrm{X}_{\Omega}\right), \preceq\right)$ and a decreasing soft operator $\mathrm{d}:\left(\mathrm{S}\left(\mathrm{X}_{\Omega}\right), \preceq\right) \rightarrow\left(\mathrm{S}\left(\mathrm{X}_{\Omega}\right), \preceq\right)$ are defined as follows: For each soft subset $\mathrm{G}_{\Omega}$ of $\mathrm{S}\left(\mathrm{X}_{\Omega}\right)$

1. $\mathfrak{i}\left(\mathrm{G}_{\Omega}\right)=(\mathfrak{i G})_{\Omega}$, where a mapping $\mathrm{iG}$ of $\Omega$ into $2^{\mathrm{X}}$ given by $\mathrm{iG}(\omega)=$ $i(G(\omega))=\{v \in X: y \preceq v$ for some $y \in G(\omega)\}$.

2. $\mathrm{d}\left(\mathrm{G}_{\Omega}\right)=(\mathrm{dG})_{\Omega}$, where a mapping $\mathrm{dG}$ of $\Omega$ into $2^{\mathrm{X}}$ given by $\mathrm{dG}(\omega)=$ $\mathrm{d}(\mathrm{G}(\omega))=\{v \in X: v \preceq y$ for some $\mathrm{y} \in \mathrm{G}(\omega)\}$.

Definition 10 [16] A soft subset $\mathrm{G}_{\Omega}$ of $(\mathrm{X}, \Omega, \preceq)$ is said to be increasing (resp. decreasing) if $\mathrm{G}_{\Omega}=\mathrm{i}\left(\mathrm{G}_{\Omega}\right)\left(\right.$ resp. $\mathrm{G}_{\Omega}=\mathrm{d}\left(\mathrm{G}_{\Omega}\right)$ ).

Theorem 1 [16] If $\mathrm{f}_{\phi}:\left(\mathrm{S}\left(\mathrm{X}_{\Omega}\right), \preceq_{1}\right) \rightarrow\left(\mathrm{S}\left(\mathrm{Y}_{\Gamma}\right), \preceq_{2}\right)$ is surjective and increasing (resp. decreasing), then the inverse image of each increasing (resp. decreasing) soft set is increasing (resp. decreasing).

\subsection{Soft topologies}

Definition 11 [45] A sub-collection $\tau$ of $\mathrm{S}\left(\mathrm{X}_{\Omega}\right)$ is called a soft topology on $\mathrm{X}$ provided that it is closed under finite soft intersection and arbitrary soft union.

By a soft topological space we mean a triple $(X, \tau, \Omega)$. Every member of $\tau$ is called soft open and its relative complement is called soft closed.

Proposition $2[45]$ In $(X, \tau, \Omega)$, a class $\tau_{\gamma}=\left\{\mathrm{G}(\omega): \mathrm{G}_{\Omega} \in \tau\right\}$ defines a classical topology on $\mathrm{X}$ for each $\omega \in \Omega$.

Proposition $3[42]$ A class $\tau^{\star}=\left\{\mathrm{G}_{\Omega}: \mathrm{G}(\omega) \in \tau_{\gamma}\right.$ for each $\left.\omega \in \Omega\right\}$ defines a soft topology on $\mathrm{X}$ finer than $\tau$.

Henceforward, $\tau^{\star}$ is called an extended soft topology.

Definition 12 [24, 37] A soft subset $\mathrm{H}_{\Omega}$ of $(\mathrm{X}, \tau, \Omega)$ which satisfies $\mathrm{H}_{\Omega} \widetilde{\subseteq} \mathrm{cl}$ $\left(\operatorname{int}\left(\mathrm{H}_{\Omega}\right)\right.$ ) is said to be soft semi open. The relative complement of a soft semi open set is said to be soft semi closed. 
Definition $13[24,37,45]$ We associate a subset $\mathrm{H}_{\Omega}$ of $(\mathrm{X}, \tau, \Omega)$ with the following four operators:

(i) $\operatorname{int}\left(\mathrm{H}_{\Omega}\right)$ (resp. int $\left.\mathrm{s}_{\mathrm{s}}\left(\mathrm{H}_{\Omega}\right)\right)$ is the largest soft open (resp. soft semi open) set contained in $\mathrm{H}_{\Omega}$.

(ii) $\operatorname{cl}\left(\mathrm{H}_{\Omega}\right)\left(\right.$ resp. $\left.\mathrm{cl}_{\mathrm{s}}\left(\mathrm{H}_{\Omega}\right)\right)$ is the smallest soft closed (resp. soft semi closed) set containing $\mathrm{H}_{\Omega}$.

Definition $14[24] f_{\phi}:(X, \tau, A) \rightarrow(Y, \theta, B)$ is said to be:

(i) soft semi continuous if $\mathrm{f}_{\phi}^{-1}\left(\mathrm{G}_{\mathrm{B}}\right)$ is soft semi open for each $\mathrm{G}_{\mathrm{B}} \in \theta$.

(ii) soft semi open (resp. soft semi closed) if $\mathrm{f}_{\phi}\left(\mathrm{U}_{A}\right)$ is soft semi open (resp. soft semi closed) for each $\mathrm{U}_{\AA}\left(\right.$ resp. $\left.\mathrm{U}_{A}^{c}\right) \in \tau$.

(iii) a soft semi homeomorphism if it is bijective, soft semi continuous and soft semi open.

Definition 15 [16] We call a quadrable system $(X, \tau, \Omega, \preceq)$ a soft topological ordered space provided that $\tau$ is a soft topology and $\preceq$ is a partially ordered set on $\mathrm{X}$.

Henceforward, we use the two notations $\left(X, \tau, \Omega, \preceq_{1}\right)$ and $\left(Y, \theta, \Gamma, \preceq_{2}\right)$ to denote soft topological ordered spaces.

Definition 16 [17] The composition of $\mathrm{f}_{\phi}:\left(\mathrm{X}, \tau, \Omega, \preceq_{1}\right) \rightarrow\left(\mathrm{Y}, \theta, \Gamma, \preceq_{2}\right)$ and $\mathrm{g}_{\lambda}:\left(\mathrm{Y}, \theta, \Gamma, \preceq_{2}\right) \rightarrow\left(\mathrm{Z}, \mathrm{v}, \mathrm{K}, \preceq_{3}\right)$ is a soft map $\mathrm{f}_{\phi} \circ \mathrm{g}_{\lambda}:\left(\mathrm{X}, \tau, \Omega, \preceq_{1}\right) \rightarrow\left(\mathrm{Z}, \mathrm{v}, \mathrm{K}, \preceq_{3}\right)$ and is given by $\left(\mathrm{f}_{\phi} \circ \mathrm{g}_{\lambda}\right)\left(\mathrm{P}_{\omega}^{\mathrm{x}}\right)=\mathrm{f}_{\phi}\left(\mathrm{g}_{\lambda}\left(\mathrm{P}_{\omega}^{\mathrm{x}}\right)\right)$.

Definition 17 [43] A map g from $\left(\mathrm{X}, \tau, \preceq_{1}\right)$ to $\left(\mathrm{Y}, \theta, \preceq_{2}\right)$ is said to be:

(i) $D$ (resp. I, B) -semi continuous if $\mathrm{g}^{-1}(\mathrm{G})$ is $D$ (resp. I, B) -semi open for each $\mathrm{G} \in \theta$.

(ii) $D($ resp. I, B) -semi open if $\mathrm{g}(\mathrm{F})$ is $D($ resp. I, B) -semi open for each $\mathrm{F} \in \tau$.

(iii) $D($ resp. I, B) -semi closed if $\mathrm{g}(\mathrm{H})$ is $D($ resp. I, B) -semi closed for each $\mathrm{F}^{\mathrm{c}} \in \tau$.

(iv) $D$ (resp. I, B) -semi homeomorphism if it is bijective, $D$ (resp. I, B) -semi continuous and D (resp. I, B) -semi open. 


\section{New types of soft semi ordered maps}

\subsection{Soft D(I, B)-semi continuity}

This subsection introduces the concepts of $\mathrm{D}(\mathrm{I}, \mathrm{B})$-semi continuity at soft point and ordinary point, where D, I and B denote "Decreasing", "Increasing" and "Balancing", respectively. We also give the equivalent terms for each one of these concepts at the ordinary points and provide some illustrative examples.

Definition 18 A soft subset $\mathrm{H}_{\Omega}$ of $\left(X, \tau, \Omega, \preceq_{1}\right)$ which is:

(i) soft semi open and increasing (resp. decreasing, balancing) is said to be SI (resp. SD, SB) -semi open.

(ii) soft semi closed and increasing (resp. decreasing, balancing) is said to be SI (resp. SD, SB) -semi closed.

Definition $19 \mathrm{f}_{\phi}:\left(\mathrm{X}, \tau, \Omega, \preceq_{1}\right) \rightarrow\left(\mathrm{Y}, \theta, \Gamma, \preceq_{2}\right)$ is called:

1. $S I$ (resp. SD, SB) -semi continuous at $\mathrm{P}_{\omega}^{\mathrm{x}} \in \widetilde{\mathrm{X}}$ if for each soft open set $\mathrm{H}_{\Gamma}$ containing $\mathrm{f}_{\phi}\left(\mathrm{P}_{\omega}^{\mathrm{x}}\right)$, there exists an $S I$ (resp. SD, SB) -semi open set $\mathrm{G}_{\Omega}$ containing $\mathrm{P}_{\omega}^{\mathrm{x}}$ such that $\mathrm{f}_{\phi}\left(\mathrm{G}_{\Omega}\right) \widetilde{\widetilde{C}} \mathrm{H}_{\Gamma}$.

2. $S I$ (resp. $S D, S B)$-semi continuous at $\mathrm{x} \in \mathrm{X}$ if it is $S I$ (resp. SD, SB) -semi continuous at each $\mathrm{P}_{\omega}^{\mathrm{x}}$.

3. $S I$ (resp. SD, SB) -semi continuous if it is $S I$ (resp. SD, SB)-semi continuous at each $\mathrm{x} \in \mathrm{X}$.

Theorem $2 \mathrm{f}_{\phi}:\left(\mathrm{X}, \tau, \Omega, \preceq_{1}\right) \rightarrow\left(\mathrm{Y}, \theta, \Gamma, \preceq_{2}\right)$ is SI (resp. SD, SB) -semi continuous iff the inverse image of each soft open set is $S I$ (resp. SD, SB) -semi open.

Proof. When $f_{\phi}$ is SD-semi continuous.

Necessity: Let $G_{\Gamma} \in \theta$. Without loss of generality, consider $f^{-1}\left(G_{\Gamma}\right) \neq \widetilde{\emptyset}$. By choosing $P_{\omega}^{x} \in X$ s.t. $P_{\omega}^{x} \in f_{\phi}^{-1}\left(G_{\Gamma}\right)$, we obtain $f_{\phi}\left(P_{\omega}^{x}\right) \in G_{\Gamma}$. Then there is an SD-semi open set $\mathrm{H}_{\Omega}$ containing $P_{\omega}^{x}$ s.t. $f_{\phi}\left(H_{\Omega}\right) \widetilde{\subseteq} G_{\Gamma}$. Since $P_{\omega}^{x}$ is chosen arbitrary, then $f_{\phi}^{-1}\left(G_{\Gamma}\right)=\widetilde{U}_{P_{\omega}^{x} \in f_{\phi}^{-1}\left(G_{\Gamma}\right)} H_{\Omega}$; therefore, $f_{\phi}^{-1}\left(G_{\Gamma}\right)$ is an SD-semi open set.

Sufficiency: Let $G_{\Gamma} \in \theta$ such that $f_{\phi}\left(P_{\omega}^{x}\right) \in \theta$. Then $P_{\omega}^{x} \in f_{\phi}^{-1}\left(G_{\Gamma}\right)$. By hypothesis, $f_{\phi}^{-1}\left(G_{\Gamma}\right)$ is an SD-semi open set. Since $f_{\phi}\left(f_{\phi}^{-1}\left(G_{\Gamma}\right)\right) \widetilde{\widetilde{C}} G_{\Gamma}$, then $f_{\phi}$ is an SD-semi continuous map at $P_{\omega}^{x}$ and since $P_{\omega}^{x}$ is selected randomly, then $f_{\phi}$ is an SD-semi continuous map. 
Remark 2 It can be seen from Definition (19) the following.

1. Every SI (SD, SB) -semi continuous map is soft semi continuous.

2. Every SB-semi continuous map is SI (SD) -semi continuous.

Examples given below manifest that the two results of the remark above are not reversible.

Example 1 Let $\Omega=\left\{\omega_{1}, \omega_{2}\right\}$ be a parameters set and $X=\{1,2,3,4\}$ be a universe set and consider $\phi: \Omega \rightarrow \Omega$ and $\mathrm{f}: \mathrm{X} \rightarrow \mathrm{X}$ are two identity maps. Let $\preceq=\triangle \bigcup\{(1,3)\}$ be a partial order relation on $X$ and consider $\tau=$ $\left\{\widetilde{\emptyset}, \widetilde{X}, F_{\Omega} \mathrm{G}_{\Omega}\right\}$ and $\theta=\left\{\widetilde{\emptyset}, \widetilde{\mathrm{Y}}, \mathrm{H}_{\Omega} \mathrm{L}_{\Omega}\right\}$ are two soft topologies on $\mathrm{X}$, where $\mathrm{F}_{\Omega}=$ $\left\{\left(\omega_{1},\{1\}\right),\left(\omega_{2},\{3,4\}\right)\right\}, \mathrm{G}_{\Omega}=\left\{\left(\omega_{1}, \emptyset\right),\left(\omega_{2},\{3\}\right)\right\}, \mathrm{H}_{\Omega}=\left\{\left(\omega_{1},\{1\}\right),\left(\omega_{2},\{2,3\}\right)\right\}$ and $\mathrm{L}_{\Omega}=\left\{\left(\omega_{1},\{1\}\right),\left(\omega_{2},\{3\}\right)\right\}$. For a soft map $\mathrm{f}_{\phi}:(\mathrm{X}, \tau, \Omega, \preceq) \rightarrow(\mathrm{X}, \theta, \Omega, \preceq)$, we note that $\mathrm{f}_{\phi}^{-1}\left(\mathrm{H}_{\Omega}\right)=\mathrm{H}_{\Omega}$ and $\mathrm{f}_{\phi}^{-1}\left(\mathrm{~L}_{\Omega}\right)=\mathrm{L}_{\Omega}$ are soft semi open sets. So $\mathrm{f}_{\phi}$ is a soft semi continuous map. But, $\mathrm{f}_{\phi}^{-1}\left(\mathrm{H}_{\Omega}\right)$ is neither an SD-semi open set nor an SI-semi open set. Hence $\mathrm{f}_{\phi}$ is not SI (SD, SB)-semi continuous.

Example 2 By replacing a partial order relation (in the above example) by $\preceq=\triangle \bigcup\{(2,4)\}($ resp. $\preceq=\triangle \bigcup\{(4,1)\})$, we obtain a soft map $\mathrm{f}_{\phi}$ is SD-semi continuous (resp. SI-continuous), but is not SB-semi continuous.

Definition 20 For any set $\mathrm{H}_{\Omega}$ in $(X, \tau, \Omega, \preceq)$, we introduce the next operators:

(i) $\mathrm{H}_{\Omega}^{\text {iso }}\left(\right.$ resp. $\left.\mathrm{H}_{\Omega}^{\mathrm{d} s o}, \mathrm{H}_{\Omega}^{\text {bso }}\right)$ is the largest $S I$ (resp. SD, SB) -semi open set contained in $\mathrm{H}_{\Omega}$.

(ii) $\mathrm{H}_{\Omega}^{\mathrm{iscl}}\left(\right.$ resp. $\left.\mathrm{H}_{\Omega}^{\mathrm{dscl}}, \mathrm{H}_{\Omega}^{\mathrm{bscl}}\right)$ is the smallest $S I$ (resp. SD, SB) -semi closed set containing $\mathrm{H}_{\Omega}$.

Lemma 1 The next properties are satisfied for a set $\mathrm{H}_{\Omega}$ in $(X, \tau, \Omega, \preceq)$.

(i) $\left(\mathrm{H}_{\Omega}^{\mathrm{dscl}}\right)^{\mathrm{c}}=\left(\mathrm{H}_{\Omega}^{\mathrm{c}}\right)^{\text {iso }}$.

(ii) $\left(H_{\Omega}^{i s c l}\right)^{c}=\left(H_{\Omega}^{c}\right)^{\text {dso }}$.

(iii) $\left(H_{\Omega}^{\text {bscl }}\right)^{c}=\left(H_{\Omega}^{c}\right)^{\text {bso }}$. 


\section{Proof.}

(i) $\left(\mathrm{H}_{\Omega}^{\mathrm{dscl}}\right)^{\mathrm{c}}=\left\{\widetilde{U} \mathrm{~F}_{\Omega}: \mathrm{F}_{\Omega} \text { is an SD-semi closed set containing } \mathrm{H}_{\Omega}\right\}^{\mathrm{c}}$ $\left(\mathrm{H}_{\Omega}^{c}\right)^{\text {iso }}$. $=\widetilde{\bigcap}\left\{\mathrm{F}_{\Omega}^{c}: \mathrm{F}_{\Omega}^{c}\right.$ is an SI-semi open set contained in $\left.\mathrm{H}_{\Omega}^{c}\right\}=$ By analogy with (i), one can prove (ii) and (iii).

Theorem 3 The next properties of $\mathrm{f}_{\phi}:\left(\mathrm{X}, \tau, \Omega, \preceq_{1}\right) \rightarrow\left(\mathrm{Y}, \theta, \Gamma, \preceq_{2}\right)$ are equivalent:

1. $\mathrm{f}_{\phi}$ is SI-semi continuous;

2. $\mathrm{f}_{\phi}^{-1}\left(\mathrm{~L}_{\Gamma}\right)$ is an SD-semi closed subset of $\widetilde{\mathrm{X}}$ for any soft closed set $\mathrm{L}_{\Gamma}$ in $\widetilde{\mathrm{Y}}$;

3. $\left(f_{\phi}^{-1}\left(M_{\Gamma}\right)\right)^{d s c l} \widetilde{\subseteq} f_{\phi}^{-1}\left(\operatorname{cl}\left(M_{\Gamma}\right)\right)$ for every $M_{\Gamma} \widetilde{\subseteq} \widetilde{Y}$;

4. $\mathrm{f}_{\phi}\left(\mathrm{N}_{\Omega}^{\mathrm{dscl}}\right) \widetilde{\widetilde{\subseteq}} \operatorname{cl}\left(\mathrm{f}_{\phi}\left(\mathrm{N}_{\Omega}\right)\right)$ for every $\mathrm{N}_{\Omega} \widetilde{\widetilde{C}} \widetilde{\mathrm{X}}$

5. $f_{\phi}^{-1}\left(\operatorname{int}\left(M_{\Gamma}\right)\right) \widetilde{\subseteq}\left(f_{\phi}^{-1}\left(M_{\Gamma}\right)\right)^{\text {iso }}$ for every $M_{\Gamma} \widetilde{\widetilde{Y}}$.

Proof. $1 \Rightarrow 2$ : Suppose $\mathrm{L}_{\Gamma}$ is a soft closed set in $\widetilde{Y}$. Then, $\mathrm{f}_{\phi}^{-1}\left(\mathrm{~L}_{\Gamma}^{\mathrm{c}}\right)$ is an SI-semi open set in $\widetilde{X}$. Now, $f_{\phi}^{-1}\left(L_{\Gamma}^{c}\right)=\left(f_{\phi}^{-1}\left(L_{\Gamma}\right)\right)^{c}$; hence, $f_{\phi}^{-1}\left(L_{\Gamma}\right)$ is an SD-semi closed set.

$2 \Rightarrow 3$ : It comes from 2 that $f_{\phi}^{-1}\left(\operatorname{cl}\left(M_{\Omega}\right)\right)$ is an SD-semi closed set in $\widetilde{X}$ for any $M_{\Gamma} \widetilde{\widetilde{Y}}$. Therefore, $\left(f_{\phi}^{-1}\left(M_{\Gamma}\right)\right)^{\mathrm{dscl}} \widetilde{\subseteq}\left(f_{\phi}^{-1}\left(\operatorname{cl}\left(M_{\Gamma}\right)\right)^{\mathrm{dscl}}=f_{\phi}^{-1}\left(\operatorname{cl}\left(M_{\Gamma}\right)\right)\right.$.

$3 \Rightarrow 4$ : We know that that $\mathrm{N}_{\Omega}^{\mathrm{dscl}} \widetilde{\subseteq}\left(\mathrm{f}_{\phi}^{-1}\left(\mathrm{f}_{\phi}\left(\mathrm{N}_{\Omega}\right)\right)^{\mathrm{dscl}}\right.$; according to 3 we have $\left(f_{\phi}^{-1}\left(f_{\phi}\left(N_{\Omega}\right)\right)^{d s c l} \widetilde{\subseteq} f_{\phi}^{-1}\left(\operatorname{cl}\left(f_{\phi}\left(N_{\Omega}\right)\right)\right.\right.$. Hence, $f_{\phi}\left(N_{\Omega}^{d s c l}\right) \widetilde{\subseteq} c l\left(f_{\phi}\left(N_{\Omega}\right)\right)$.

$4 \Rightarrow 5$ : For any soft set $M_{\Gamma}$ in $\widetilde{Y}$, we obtain from Lemma (1) that $f_{\phi}\left(\widetilde{X}-\left(f_{\phi}^{-1}\right.\right.$ $\left.\left.\left(N_{\Omega}\right)\right)^{i s o}\right)=f_{\phi}\left(\left(\left(f_{\phi}^{-1}\left(N_{\Omega}\right)\right)^{c}\right)^{d s c l}\right)$. It follows from statement 4 , that $f_{\phi}\left(\left(\left(f_{\phi}^{-1}\right.\right.\right.$ $\left.\left.\left.\left(\mathrm{N}_{\Omega}\right)\right)^{\mathrm{c}}\right)^{\mathrm{dscl}}\right) \widetilde{\widetilde{C}} \operatorname{cl}\left(f_{\phi}\left(f_{\phi}^{-1}\left(\mathrm{~N}_{\Omega}\right)\right)^{c}\right)=\operatorname{cl}\left(f_{\phi}\left(f_{\phi}^{-1}\left(N_{\Omega}^{c}\right)\right)\right) \widetilde{\subseteq} \operatorname{cl}\left(\widetilde{Y}-N_{\Omega}\right)=\widetilde{Y}-i n t\left(N_{\Omega}\right)$. Therefore $\left(\widetilde{X}-\left(f_{\phi}^{-1}\left(N_{\Omega}\right)\right)^{i s o}\right) \widetilde{\widetilde{C}} f_{\phi}^{-1}\left(\widetilde{Y}-\operatorname{int}\left(N_{\Omega}\right)\right)=\widetilde{X}-f_{\phi}^{-1}\left(\operatorname{int}\left(N_{\Omega}\right)\right)$. Thus $f_{\phi}^{-1}\left(\operatorname{int}\left(N_{\Omega}\right)\right) \widetilde{\widetilde{C}}\left(f_{\phi}^{-1}\left(N_{\Omega}\right)\right)^{\text {iso }}$.

$5 \Rightarrow 1$ : Consider $M_{\Gamma}$ is a soft open set in $\widetilde{Y}$. Then $f_{\phi}^{-1}\left(M_{\Gamma}\right)=f_{\phi}^{-1}\left(\operatorname{int}\left(M_{\Gamma}\right)\right) \widetilde{\subseteq}$

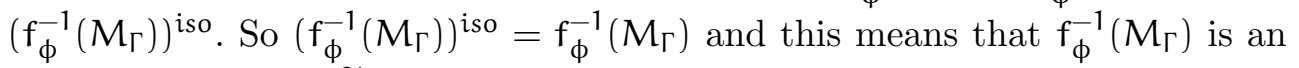
SI-semi open set in $\widetilde{X}$.

Theorem 4 The next properties of $\mathrm{f}_{\phi}:\left(\mathrm{X}, \tau, \Omega, \preceq_{1}\right) \rightarrow\left(\mathrm{Y}, \theta, \Gamma, \preceq_{2}\right)$ are equivalent: 
1. $\mathrm{f}_{\phi}$ is SD-semi continuous (resp. SB-semi continuous);

2. $\mathrm{f}_{\phi}^{-1}\left(\mathrm{~L}_{\Gamma}\right)$ is an SI-semi closed (resp. SB-semi closed) set in $\widetilde{\mathrm{X}}$ for each soft closed set $\mathrm{L}_{\Gamma}$ in $\widetilde{\mathrm{Y}}$

3. $\left(f_{\phi}^{-1}\left(M_{\Gamma}\right)\right)^{i s c l} \widetilde{\simeq} f_{\phi}^{-1}\left(\operatorname{cl}\left(M_{\Gamma}\right)\right)\left(\right.$ resp. $\left.\left(f_{\phi}^{-1}\left(M_{\Gamma}\right)\right)^{\text {bscl }} \widetilde{\simeq} f_{\phi}^{-1}\left(\operatorname{cl}\left(M_{\Gamma}\right)\right)\right)$ for every $\mathrm{M}_{\Gamma} \widetilde{\widetilde{Y}} \widetilde{Y}$

4. $\mathrm{f}_{\phi}\left(\mathrm{N}_{\Omega}^{\mathrm{iscl}}\right) \widetilde{\widetilde{\subseteq}} \operatorname{cl}\left(\mathrm{f}_{\phi}\left(\mathrm{N}_{\Omega}\right)\right)$ (resp. $\left.\mathrm{f}_{\phi}\left(\mathrm{N}_{\Omega}^{\mathrm{bscl}}\right) \widetilde{\widetilde{\subseteq}} \operatorname{cl}\left(\mathrm{f}_{\phi}\left(\mathrm{N}_{\Omega}\right)\right)\right)$ for every $\mathrm{N}_{\Omega} \widetilde{\subseteq} \widetilde{\mathrm{X}}$;

5. $f_{\phi}^{-1}\left(\operatorname{int}\left(M_{\Gamma}\right)\right) \widetilde{\widetilde{C}}\left(f_{\phi}^{-1}\left(M_{\Gamma}\right)\right)^{\mathrm{dso}}\left(\right.$ resp. $\left.f_{\phi}^{-1}\left(\operatorname{int}\left(M_{\Gamma}\right)\right) \widetilde{\subseteq}\left(f_{\phi}^{-1}\left(M_{\Gamma}\right)\right)^{b s o}\right)$ for ev$\operatorname{ery} \mathrm{M}_{\Gamma} \widetilde{\widetilde{Y}}$.

Proof. Similar to the proof of Theorem (3).

Theorem 5 Let a soft topology $\tau^{\star}$ be extended. Then $\mathrm{g}_{\phi}:\left(X, \tau^{\star}, \Omega, \preceq_{1}\right) \rightarrow$ $\left(\mathrm{Y}, \theta, \Gamma, \preceq_{2}\right)$ is $S I$ (resp. SD, SB) -semi continuous iff a crisp map $\mathrm{g}:\left(\mathrm{X}, \tau_{\gamma}^{\star}, \preceq_{1}\right.$ )$\rightarrow\left(\mathrm{Y}, \theta_{\phi(\omega)}, \preceq_{2}\right)$ is I (resp. D, B) -semi continuous.

Proof. $\Rightarrow$ : Consider $U$ is an open set in $\left(Y, \theta_{\phi(\omega)}, \preceq_{2}\right)$. Then there is a soft open set $\mathrm{G}_{\Gamma}$ in $\left(\mathrm{Y}, \theta, \Gamma, \preceq_{2}\right)$ s.t. $\mathrm{G}(\phi(\omega))=\mathrm{U}$. Since $\mathrm{g}_{\phi}$ is an SI (resp. SD, SB) -semi continuous map, then $g_{\phi}^{-1}\left(G_{\Gamma}\right)$ is an SI (resp. SD, SB) -semi open set. From Definition (5), a soft set $g_{\phi}^{-1}\left(G_{\Gamma}\right)=\left(g_{\phi}^{-1}(G)\right)_{\Omega}$ in $\left(X, \tau, \Omega, \preceq_{1}\right)$ is given by $g_{\phi}^{-1}(G)(\omega)=g^{-1}(G(\phi(\omega)))$ for any $\omega \in \Omega$. Now, $\tau^{\star}$ is extended; thus, a set $g^{-1}(G(\phi(\omega)))=g^{-1}(U)$ in $\left(X, \tau_{\gamma}, \preceq_{1}\right)$ is I (resp. D, B) -semi open. This proves that $\mathrm{g}$ is $\mathrm{I}$ (resp. $\mathrm{D}, \mathrm{B}$ ) -semi continuous.

$\Leftarrow$ : Consider $G_{\Gamma}$ is a soft open set in $\left(Y, \theta, \Gamma, \preceq_{2}\right)$. Then a soft set $g_{\phi}^{-1}\left(G_{\Gamma}\right)=$ $\left(g_{\phi}^{-1}(G)\right)_{\Omega}$ in $\left(X, \tau^{\star}, \Omega, \preceq_{1}\right)$ isgiven by $g_{\phi}^{-1}(G)(\omega)=g^{-1}(G(\phi(\omega)))$ for any $\omega \in \Omega$. Since amap g is I (resp. D, B) -semi continuous, a set $g^{-1}(G(\phi(\omega)))$ in $\left(X, \tau_{\gamma}^{\star}, \preceq_{1}\right)$ is I (resp. D, B) -semi open. Now, $\tau^{\star}$ is extended; thus, $g_{\phi}^{-1}\left(G_{\Gamma}\right)$ is an SI (resp. SD, SB) -semi open set in $\left(X, \tau^{\star}, \Omega, \preceq_{1}\right)$. This proves that a soft map $g_{\phi}$ is SI (resp. SD, SB) -semi continuous.

Proposition 4 Let $\mathrm{f}_{\phi}:\left(\mathrm{X}, \tau, \Omega, \preceq_{1}\right) \rightarrow\left(\mathrm{Y}, \theta, \Gamma, \preceq_{2}\right)$ be SB-semi continuous and surjective. If $\preceq_{1}$ is linearly ordered, then $\theta$ is the indiscrete soft topology.

\subsection{Soft $I(D, B)$-semi open and soft $I(D, B)$-semi closed maps}

In the following part, we present the notions of soft I(D, B)-semi open and soft $\mathrm{I}(\mathrm{D}, \mathrm{B})$-semi closed maps. Then, we elucidate the relationships among them 
with the help of examples. Finally, we characterize each one of these concepts and study some properties.

Definition $21 f_{\phi}:\left(X, \tau, \Omega, \preceq_{1}\right) \rightarrow\left(Y, \tau, \Gamma, \preceq_{2}\right)$ is called:

(i) $S I$ (resp. SD, SB) -semi open if the image of any soft open set in $\widetilde{\mathrm{X}}$ is an SI (resp. SD, SB) -semi open set in $\widetilde{Y}$.

(ii) $S I$ (resp. SD, SB) -semi closed if the image of any soft closed set in $\widetilde{\mathrm{X}}$ is an $S I$ (resp. SD, SB) -semi closed set in $\widetilde{Y}$.

Remark 3 Note that:

1. an $S I(S D, S B)$-semi open map is soft semi open.

2. an $S I(S D, S B)$-semi closed map is soft semi closed.

3. an $S B$-semi open (resp. SB-semi closed) map is SI (SD) -semi open (resp. $S I(S D)$-semi closed).

Examples given below manifest that the three results of the remark above are not reversible.

Example 3 Let $\Omega, X, \phi: \Omega \rightarrow \Omega, f: X \rightarrow X$ and $\preceq$ be the same as in Example (1). Consider $\tau=\left\{\widetilde{\emptyset}, \widetilde{X}, F_{\Omega}\right\}$ and $\theta=\left\{\widetilde{\emptyset}, \widetilde{Y}, \mathrm{~L}_{\Omega}\right\}$ are two soft topologies on $\mathrm{X}$, where $\mathrm{F}_{\Omega}=\left\{\left(\omega_{1},\{1\}\right),\left(\omega_{2},\{3,4\}\right)\right\}$ and $\mathrm{L}_{\Omega}=\left\{\left(\omega_{1},\{1\}\right),\left(\omega_{2},\{3\}\right)\right\}$. For a soft map $\mathrm{f}_{\phi}:(\mathrm{X}, \tau, \Omega, \preceq) \rightarrow(\mathrm{X}, \theta, \Omega, \preceq)$, we note that $\mathrm{f}_{\phi}\left(\mathrm{F}_{\Omega}\right)=\mathrm{F}_{\Omega}$ is a soft semi open set. So $\mathrm{f}_{\phi}$ is a soft semi open map. On the other hand, $\mathrm{f}_{\phi}\left(\mathrm{F}_{\Omega}\right)$ is neither an SD-semi open set nor an SI-semi open set. Hence $\mathrm{f}_{\phi}$ is not SI (SD, SB)-semi open. Also, $\mathrm{f}_{\phi}$ is a soft semi closed map, but it is not SI (SD, SB)-semi closed.

Example 4 By replacing a partial order relation (given in the example above) by $\preceq=\triangle \bigcup\{(2,4)\}$, we obtain $\boldsymbol{f}_{\phi}$ is SI-semi open and SD-semi closed, but it is neither an SB-semi open map nor an SB-semi closed map. Also, if we replace only the partial order relation by $\preceq=\triangle \bigcup\{(1,2)\}$, then the soft map $\mathrm{f}_{\phi}$ is SD-open and SI-semi closed, but it is neither an SB-semi open map nor an SB-semi closed map.

Theorem 6 The next properties of $\mathrm{f}_{\phi}:\left(X, \tau, \Omega, \preceq_{1}\right) \rightarrow\left(\mathrm{Y}, \theta, \Gamma, \preceq_{2}\right)$ are equivalent:

1. $\mathrm{f}_{\phi}$ is SI-semi open; 

2. $\operatorname{int}\left(f_{\phi}^{-1}\left(M_{\Gamma}\right)\right) \widetilde{\subseteq} f_{\phi}^{-1}\left(M_{\Gamma}^{i s o}\right)$ for any $M_{\Gamma} \widetilde{\widetilde{Y}} \widetilde{Y}$
3. $\mathrm{f}_{\phi}\left(\operatorname{int}\left(\mathrm{N}_{\Omega}\right)\right) \widetilde{\subseteq}\left(\mathrm{f}_{\phi}\left(\mathrm{N}_{\Omega}\right)\right)^{\text {iso }}$ for any $\mathrm{N}_{\Omega} \widetilde{\subseteq} \widetilde{\mathrm{X}}$.

Proof. $1 \Rightarrow 2$ : It is clear that $\operatorname{int}\left(f_{\phi}^{-1}\left(M_{\Gamma}\right)\right)$ is a soft open set in $\widetilde{X}$ for any soft set $M_{\Gamma}$ in $\widetilde{Y}$. Now, $f_{\phi}\left(\operatorname{int}\left(f_{\phi}^{-1}\left(M_{\Gamma}\right)\right)\right)$ is an SI-semi open set in $\widetilde{Y}$. Since $f_{\phi}\left(\operatorname{int}\left(f_{\phi}^{-1}\left(M_{\Gamma}\right)\right)\right) \widetilde{\widetilde{\subseteq}} f_{\phi}\left(f_{\phi}^{-1}\left(M_{\Gamma}\right)\right) \widetilde{\widetilde{\subseteq}} M_{\Gamma}$, then $\operatorname{int}\left(f_{\phi}^{-1}\left(M_{\Gamma}\right)\right) \widetilde{\widetilde{\subseteq}} f_{\phi}^{-1}\left(M_{\Gamma}^{\text {iso }}\right)$.

$2 \Rightarrow 3$ : Given a soft set $N_{\Omega}$ in $\widetilde{X}$, according to $2 \operatorname{int}\left(f_{\phi}^{-1}\left(f_{\phi}\left(N_{\Omega}\right)\right)\right) \widetilde{\subseteq} f_{\phi}^{-1}\left(\left(f_{\phi}\right.\right.$ $\left.\left.\left(N_{\Omega}\right)\right)^{i s o}\right)$. Since $\operatorname{int}\left(N_{\Omega}\right) \widetilde{\subseteq} f_{\phi}^{-1}\left(f_{\phi}\left(\operatorname{int}\left(f_{\phi}^{-1}\left(f_{\phi}\left(N_{\Omega}\right)\right)\right)\right)\right) \widetilde{\subseteq} f_{\phi}^{-1}\left(\left(f_{\phi}\left(N_{\Omega}\right)\right)^{\text {iso }}\right)$, then $f_{\phi}\left(\operatorname{int}\left(N_{\Omega}\right)\right) \widetilde{\subseteq}\left(f_{\phi}\left(N_{\Omega}\right)\right)^{\text {iso }}$.

$3 \Rightarrow 1$ : Let $G_{\Omega}$ be a soft open set in $\widetilde{X}$. Then $f_{\phi}\left(\operatorname{int}\left(G_{\Omega}\right)\right)=f_{\phi}\left(G_{\Omega}\right) \widetilde{\subseteq}\left(f_{\phi}\left(G_{\Omega}\right)\right)^{\text {iso }}$. Hence, $f_{\phi}$ is an SI-semi open map.

Following similar technique, the following two theorems are proved.

Theorem 7 The following three properties of $f_{\phi}:\left(X, \tau, \Omega, \preceq_{1}\right) \rightarrow\left(Y, \theta, \Gamma, \preceq_{2}\right)$ are equivalent:

1. $\mathrm{f}_{\phi}$ is $S D$-semi open (resp. SB-semi open);

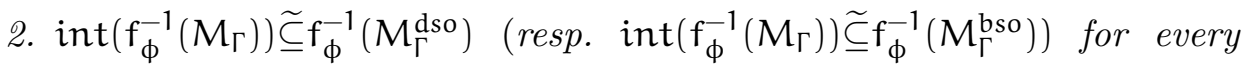
$M_{\Gamma} \widetilde{\widetilde{Y}}$

3. $\mathrm{f}_{\phi}\left(\operatorname{int}\left(\mathrm{N}_{\Omega}\right)\right) \widetilde{\widetilde{\subseteq}}\left(\mathrm{f}_{\phi}\left(\mathrm{N}_{\Omega}\right)\right)^{\mathrm{dso}}\left(\right.$ resp. $\left.\mathrm{f}_{\phi}\left(\operatorname{int}\left(\mathrm{N}_{\Omega}\right)\right) \widetilde{\subseteq}\left(\mathrm{f}_{\phi}\left(\mathrm{N}_{\Omega}\right)\right)^{\text {bso }}\right)$ for every $\mathrm{N}_{\Omega} \widetilde{\subseteq} \widetilde{\mathrm{X}}$.

Theorem 8 The next statements hold for $\mathrm{f}_{\phi}:\left(\mathrm{X}, \tau, \Omega, \preceq_{1}\right) \rightarrow\left(\mathrm{Y}, \theta, \Gamma, \preceq_{2}\right)$ :

1. $\mathrm{f}_{\phi}$ is SI-semi closed iff $\left(\mathrm{f}_{\phi}\left(\mathrm{G}_{\Omega}\right)\right)^{i s c l} \widetilde{\subseteq} \mathrm{f}_{\phi}\left(\operatorname{cl}\left(\mathrm{G}_{\Omega}\right)\right)$ for every $\mathrm{G}_{\Omega} \widetilde{\subseteq} \widetilde{\mathrm{X}}$.

2. $\mathrm{f}_{\phi}$ is SD-semi closed iff $\left(\mathrm{f}_{\phi}\left(\mathrm{G}_{\Omega}\right)\right)^{\mathrm{dscl}} \widetilde{\subseteq} \mathrm{f}_{\phi}\left(\operatorname{cl}\left(\mathrm{G}_{\Omega}\right)\right)$ for every $\mathrm{G}_{\Omega} \widetilde{\subseteq} \widetilde{\mathrm{X}}$.

3. $\mathrm{f}_{\phi}$ is SB-semi closed iff $\left(\mathrm{f}_{\phi}\left(\mathrm{G}_{\Omega}\right)\right)^{\mathrm{bscl}} \widetilde{\widetilde{f}} \mathrm{f}_{\phi}\left(\operatorname{cl}\left(\mathrm{G}_{\Omega}\right)\right)$ for every $\mathrm{G}_{\Omega} \widetilde{\subseteq} \widetilde{X}$.

Proof. We only prove 1 .

Necessity: Since $f_{\phi}$ is SI-semi closed, $f_{\phi}\left(\operatorname{cl}\left(G_{\Omega}\right)\right)$ is an SI-semi closed set in $\widetilde{Y}$ and since $f_{\phi}\left(G_{\Omega}\right) \stackrel{\widetilde{C}}{\subseteq} f_{\phi}\left(\operatorname{cl}\left(G_{\Omega}\right)\right),\left(f_{\phi}\left(G_{\Omega}\right)\right)^{i s c l} \widetilde{\simeq} f_{\phi}\left(\operatorname{cl}\left(G_{\Omega}\right)\right)$.

Sufficiency: Consider $\mathrm{H}_{\Omega}$ is a soft closed set in $\widetilde{X}$. Then $\mathrm{f}_{\phi}\left(\mathrm{H}_{\Omega}\right) \widetilde{\subseteq}\left(\mathrm{f}_{\phi}\left(\mathrm{H}_{\Omega}\right)\right)^{\text {iscl }} \widetilde{\subseteq}$ $f_{\phi}\left(\operatorname{cl}\left(H_{\Omega}\right)\right)=f_{\phi}\left(H_{\Omega}\right)$. Therefore, $f_{\phi}\left(H_{\Omega}\right)=\left(f_{\phi}\left(H_{\Omega}\right)\right)^{\text {iscl }}$. This means that $f_{\phi}\left(H_{\Omega}\right)$ is an SI-semi closed set. 
Theorem 9 The next hold for a bijective soft map $\mathrm{f}_{\phi}:\left(\mathrm{X}, \tau, \Omega, \preceq_{1}\right) \rightarrow(\mathrm{Y}, \theta$, $\left.\Gamma, \preceq_{2}\right)$ :

(i) $\mathrm{f}_{\phi}$ is $S I$ (resp. $S D, S B$ ) -semi open if and only if $\mathrm{f}_{\phi}$ is $S D$ (resp. $S D, S B$ ) -semi closed.

(ii) $\mathrm{f}_{\phi}$ is $S I$ (resp. $\left.S D, S B\right)$-semi open if and only if $\mathrm{f}_{\phi}^{-1}$ is $S I$ (resp. $S D, S B$ ) -semi continuous.

(iii) $\mathrm{f}_{\phi}$ is $S D$ (resp. SI, SB) -semi closed if and only if $\mathrm{f}_{\phi}^{-1}$ is SI (resp. SD, $S B)$-semi continuous.

Proof. We prove the cases outside the parenthesis and the cases between parenthesis can be made similarly.

(i) Necessity: Let $H_{\Omega}$ be a soft closed set in $\widetilde{X}$ and consider $f_{\phi}$ is an SIsemi open map. Then $H_{\Omega}^{c}$ is soft open and $f_{\phi}\left(H_{\Omega}^{c}\right)$ is SI-semi open. Bijectiveness of $f_{\phi}$ leads to that $f_{\phi}\left(H_{\Omega}^{c}\right)=\left[f_{\phi}\left(H_{\Omega}\right)\right]^{c}$. This automatically implies that $f_{\phi}\left(H_{\Omega}\right)$ is SD-semi closed. Thus, $f_{\phi}$ is an SD-semi closed map. Following similar technique, the sufficient condition is proved.

(ii) Necessity: Let $G_{\Omega}$ be a soft open set in $\widetilde{X}$ and consider $f_{\phi}$ is an SI-semi open map. Then $f_{\phi}\left(G_{\Omega}\right)$ is SI-semi open. Bijectiveness of $f_{\phi}$ leads to that $f_{\phi}\left(G_{\Omega}\right)=\left(f_{\phi}^{-1}\right)^{-1}\left(G_{\Omega}\right)$. This automatically implies that $\left(f_{\phi}^{-1}\right)^{-1}\left(G_{\Omega}\right)$ is SI-semi open. Thus $f_{\phi}^{-1}$ is an SI-semi continuous map. Following similar technique, the sufficient condition is proved.

(iii) It follows from (i) and (ii).

Theorem 10 Let a soft topology $\theta^{\star}$ be extended and a map $\phi$ be injective. Then $\mathrm{g}_{\phi}:\left(\mathrm{X}, \tau, \Omega, \preceq_{1}\right) \rightarrow\left(\mathrm{Y}, \theta^{\star}, \Gamma, \preceq_{2}\right)$ is $S I$ (resp. SD, SB) -semi open iff a crisp map $\mathrm{g}:\left(\mathrm{X}, \tau_{\gamma}, \preceq_{1}\right) \rightarrow\left(\mathrm{Y}, \theta_{\phi(\omega)}^{\star}, \preceq_{2}\right)$ is $I$ (resp. D, B) -semi open.

Proof. Let $U$ be an open set in $\left(X, \tau_{\gamma}, \preceq_{1}\right)$ and $\phi(\omega)=f$. Then there is a soft open set $G_{\Omega}$ in $\left(X, \tau, \Omega, \preceq_{1}\right)$ s.t. $G(\omega)=U$. Since $g_{\phi}$ is an SI (resp. SD, SB) -semi open map, then $g_{\phi}\left(G_{\Omega}\right)$ is an SI (resp. SD, SB) semi open set. Now, a soft set $g_{\phi}\left(G_{\Omega}\right)=\left(g_{\phi}(G)\right)_{\Gamma}$ in $\left(Y, \theta, \Gamma, \preceq_{2}\right)$ is given by $g_{\phi}(G)(f)=\bigcup_{\omega \in \phi^{-1}(f)} g(G(\omega))$ for each $f \in \Gamma$. View of $\theta^{\star}$ is extended, a set $\bigcup_{\omega \in \phi^{-1}(f)} g(G(\omega))=g(U)$ in $\left(Y, \theta_{\phi(\omega)}, \preceq_{2}\right)$ is I (resp. D, B) -semi open. 
Hence a map $g$ is I (resp. D, B) -semi open. Conversely, consider $G_{\Omega}$ is a soft open set in $\left(X, \tau, \Omega, \preceq_{1}\right)$. Then a soft set $g_{\phi}\left(G_{\Omega}\right)=\left(g_{\phi}(G)\right)_{\Gamma}$ in $\left(Y, \theta^{\star}, \Gamma, \preceq_{2}\right)$ is given by $g_{\phi}(G)(f)=\bigcup_{\omega \in \phi^{-1}(f)} g(G(\omega))$ for each $f \in \Gamma$. Since a map $g$ is $I$ (resp. D, B) -semi open, a set $\bigcup_{\omega \in \phi^{-1}(f)} g(G(\omega))$ in $\left(Y, \theta_{\phi(\omega)}^{\star}, \preceq_{2}\right)$ is I (resp. $\mathrm{D}, \mathrm{B})$-semi open. Now, $\theta^{\star}$ is an extended soft topology on $Y, g_{\phi}\left(G_{\Omega}\right)$ is an SI (resp. SD, SB) -semi open subset of $\left(Y, \theta^{\star}, \Gamma, \preceq_{2}\right)$. Hence a soft map $g_{\phi}$ is SI (resp. SD, SB) -semi open.

Theorem 11 Let a soft topology $\theta^{\star}$ be extended and a map $\phi$ is injective. Then $\mathrm{g}_{\phi}:\left(\mathrm{X}, \tau, \Omega, \preceq_{1}\right) \rightarrow\left(\mathrm{Y}, \theta^{\star}, \Gamma, \preceq_{2}\right)$ is $S I$ (resp. SD, SB) -semi closed iff a crisp map $\mathrm{g}:\left(\mathrm{X}, \tau_{\gamma}, \preceq_{1}\right) \rightarrow\left(\mathrm{Y}, \theta_{\phi(\omega)}^{\star}, \preceq_{2}\right)$ is I (resp. D, B) -semi closed.

Proposition 5 Let $x \in\{I, D, B\}$ and consider $f_{\phi}:\left(X, \tau, \Omega, \preceq_{1}\right) \rightarrow\left(Y, \theta, \Gamma, \preceq_{2}\right)$ and $\mathrm{g}_{\lambda}:\left(\mathrm{Y}, \theta, \Gamma, \preceq_{2}\right) \rightarrow\left(\mathrm{Z}, \mathcal{v}, \mathrm{K}, \preceq_{3}\right)$ are soft maps. Then:

(i) If $\mathrm{f}_{\phi}$ is an Sx-semi continuous map and $\mathrm{g}_{\lambda}$ is a soft continuous map, then $\mathrm{g}_{\lambda} \circ \mathrm{f}_{\phi}$ is an Sx-continuous map.

(ii) If $\mathrm{f}_{\phi}$ is a soft open (resp. soft closed) map and $\mathrm{g}_{\lambda}$ is an Sx-semi open (resp. Sx-semi closed) map, then $\mathrm{g}_{\lambda} \circ \mathrm{f}_{\phi}$ is an Sx-semi open (resp. Sxsemi closed) map.

(iii) If $\mathrm{g}_{\lambda} \circ \mathrm{f}_{\phi}$ is an Sx-open map and $\mathrm{f}_{\phi}$ is surjective soft continuous, then $\mathrm{g}_{\lambda}$ is an Sx-open map.

\subsection{Soft I(D, B)-semi homeomorphism}

We define and investigate in this subsection, the concepts of soft $I(D, B)$ semi homeomorphism maps. We discussed their main features and verify some findings related to them.

Definition 22 A bijective soft map $\mathrm{g}_{\phi}:\left(\mathrm{X}, \tau, \Omega, \preceq_{1}\right) \rightarrow\left(\mathrm{Y}, \theta, \Gamma, \preceq_{2}\right)$ is called SI (resp. SD, SB) -semi homeomorphism if it is SI-semi continuous and SIsemi open (resp. SD-semi continuous and SD-semi open, SB-semi continuous and $S B$-semi open).

Remark 4 Note that:

1. an $S I(S D, S B)$-semi homeomorphism map is soft semi homeomorphism.

2. an SB-semi homeomorphism map is SI-semi homeomorphism or SDsemi homeomorphism. 
Examples given below manifest that the results of the remark above are not reversible.

Example 5 Let $\Omega, X, \phi: \Omega \rightarrow \Omega, f: X \rightarrow X$ and $\preceq$ be the same as in Example (1). Consider $\tau=\left\{\widetilde{\emptyset}, \widetilde{X}, F_{\Omega}, \mathrm{L}_{\Omega}\right\}$ and $\theta=\left\{\widetilde{\emptyset}, \widetilde{Y}, \mathrm{~L}_{\Omega}\right\}$ are two soft topologies on $\mathrm{X}$, where $\mathrm{F}_{\Omega}=\left\{\left(\omega_{1},\{1\}\right),\left(\omega_{2},\{3,4\}\right)\right\}$ and $\mathrm{L}_{\Omega}=\left\{\left(\omega_{1},\{1\}\right),\left(\omega_{2},\{3\}\right)\right\}$. Then we find that $\mathrm{f}_{\phi}:(\mathrm{X}, \tau, \Omega, \preceq) \rightarrow(\mathrm{X}, \theta, \Omega, \preceq)$ is a soft semi homeomorphism map, but it is neither an SD-semi homeomorphism map nor an SI-semi homeomorphism map. Hence $\mathrm{f}_{\phi}$ is not SI (SD, SB)-semi homeomorphism.

Example 6 By replacing a partial order relation (given in example above) by $\preceq=\triangle \bigcup\{(2,4)\}$, we find that a soft map $\mathrm{f}_{\phi}$ is SI-semi homeomorphism, but it is not an SB-semi homeomorphism map. Also, replacing a partial order relation (given in example above) by $\preceq=\triangle \bigcup\{(1,2)\}$ leads to that a soft map $\mathrm{f}_{\phi}$ is SD-homeomorphism, but it is not an $S B$-semi homeomorphism map.

Theorem 12 Consider $\mathrm{f}_{\phi}:\left(\mathrm{X}, \tau, \Omega, \preceq_{1}\right) \rightarrow\left(\mathrm{Y}, \theta, \Gamma, \preceq_{2}\right)$ is a bijective soft map and let $(\gamma, \lambda) \in\{(\mathrm{Is}, \mathrm{dscl}),(\mathrm{Ds}, \mathrm{iscl}),(\mathrm{Bs}, \mathrm{bscl})\}$. Then $\mathrm{f}_{\phi}$ is soft $\gamma$ homeomorphism if and only if $\left(\mathrm{f}_{\phi}\left(\mathrm{G}_{\Omega}\right)\right)^{\lambda}=\mathrm{f}_{\phi}\left(\operatorname{cl}\left(\mathrm{G}_{\Omega}\right)\right)=\operatorname{cl}\left(\mathrm{f}_{\phi}\left(\mathrm{G}_{\Omega}\right)\right)=$ $\mathrm{f}_{\phi}\left(\mathrm{G}_{\Omega}^{\lambda}\right)$ for every $\mathrm{G}_{\Omega} \widetilde{\simeq} \widetilde{\mathrm{X}}$.

Proof. We only prove the case of $(\gamma, \lambda)=(\mathrm{Is}, \mathrm{dscl})$.

Necessity: The property $f_{\phi}$ is an SI-semi homeomorphism map implies that $f_{\phi}\left(G_{\Omega}^{\text {dscl }}\right) \widetilde{\widetilde{C}} c l\left(f_{\phi}\left(G_{\Omega}\right)\right)$ and $\left(f_{\phi}\left(G_{\Omega}\right)\right)^{\text {dscl }} \widetilde{\simeq} f_{\phi}\left(\operatorname{cl}\left(G_{\Omega}\right)\right)$ for every $G_{\Omega} \widetilde{\simeq} \widetilde{X}$. So $f_{\phi}$ $\left(\operatorname{cl}\left(G_{\Omega}\right)\right) \widetilde{\widetilde{\subseteq}} f_{\phi}\left(G_{\Omega}^{\text {dscl }}\right) \widetilde{\widetilde{C}} \operatorname{cl}\left(f_{\phi}\left(G_{\Omega}\right)\right) \widetilde{\widetilde{\subseteq}}\left(f_{\phi}\left(G_{\Omega}\right)\right)^{\text {dscl }}$ and $\operatorname{cl}\left(f_{\phi}\left(G_{\Omega}\right)\right) \widetilde{\widetilde{\subseteq}}\left(f_{\phi}\left(G_{\Omega}\right)\right)^{\mathrm{dscl}}$ $\widetilde{\subseteq} f_{\phi}\left(\operatorname{cl}\left(G_{\Omega}\right)\right) \widetilde{\subseteq} f_{\phi}\left(G_{\Omega}^{\text {dscl }}\right)$. By the preceding two inclusion relations, we obtain the required equality relation.

Sufficiency: The equality relation $\left(f_{\phi}\left(G_{\Omega}\right)\right)^{d s c l}=f_{\phi}\left(\operatorname{cl}\left(G_{\Omega}\right)\right)=\operatorname{cl}\left(f_{\phi}\left(G_{\Omega}\right)\right)=$ $f_{\phi}\left(G_{\Omega}^{\text {dscl }}\right)$ implies that $f_{\phi}\left(G_{\Omega}^{\text {dscl }}\right) \widetilde{\subseteq} c l\left(f_{\phi}\left(G_{\Omega}\right)\right)$ and $\left(f_{\phi}\left(G_{\Omega}\right)\right)$ dscl $\widetilde{\subseteq} f_{\phi}\left(\operatorname{cl}\left(G_{\Omega}\right)\right)$. So $f_{\phi}$ is SI-semi continuous and SD-semi closed map. Hence the desired result is proved.

Theorem 13 If a bijective soft map $\mathrm{f}_{\phi}:\left(\mathrm{X}, \tau, \Omega, \preceq_{1}\right) \rightarrow\left(\mathrm{Y}, \theta, \Gamma, \preceq_{2}\right)$ is SIsemi continuous (resp. SD-semi continuous, SB-semi continuous), Then the following three statements are equivalent:

1. $\mathrm{f}_{\phi}$ is SI-semi homeomorphism (resp. SD-semi homeomorphism, SB-semi homeomorphism);

2. $\mathrm{f}_{\phi}^{-1}$ is SI-semi continuous (resp. SD-semi continuous, SB-semi continuous); 
3. $\mathrm{f}_{\phi}$ is SD-semi closed (resp. SI-semi closed, SB-semi closed).

Proof. $1 \Rightarrow 2$ : Since $f_{\phi}$ is an SI-semi homeomorphism (resp. SD-semi homeomorphism, SB-semi homeomorphism) map, $f_{\phi}$ is SI-semi open (resp. SD-semi open, SB-semi open). It comes from item 2 of Theorem (9) that $f_{\phi}^{-1}$ is SI-semi continuous (resp. SD-semi continuous, SB-semi continuous).

$2 \Rightarrow 3$ : It comes from item 3 of Theorem (9).

$3 \Rightarrow 1$ : It suffices to prove that $f_{\phi}$ is an SI-semi open (resp. SD-semi open, SB-semi open) map. This comes from item 1 of Theorem (9).

Theorem 14 Let soft topologies $\tau^{\star}$ and $\theta^{\star}$ be extended on $\mathrm{X}$ and $\mathrm{Y}$, respectively. Then a soft map $\mathrm{g}_{\phi}:\left(\mathrm{X}, \tau^{\star}, \Omega, \preceq_{1}\right) \rightarrow\left(\mathrm{Y}, \theta^{\star}, \Gamma, \preceq_{2}\right)$ is $S I$ (resp. SD, SB) -semi homeomorphism iff amap $\mathrm{g}:\left(\mathrm{X}, \tau_{\gamma}^{\star}, \preceq_{1}\right) \rightarrow\left(\mathrm{Y}, \theta_{\phi(\omega)}^{\star}, \preceq_{2}\right)$ is I (resp. D, B) -semi homeomorphism.

Proof. Directly from Theorem (5) and Theorem (10).

Proposition 6 Let the two soft topologies $\tau$ and $\theta$ on $\mathrm{X}$ and $\mathrm{Y}$, respectively, do not belong to \{soft discrete topology, soft indiscrete topology\}. If a soft map $\mathrm{f}_{\phi}:\left(\mathrm{X}, \tau, \Omega, \preceq_{1}\right) \rightarrow\left(\mathrm{Y}, \theta, \Gamma, \preceq_{2}\right)$ is SB-semi homeomorphism, then $\preceq_{1}$ and $\preceq_{2}$ is not linearly ordered.

\section{Conclusion}

Study topological concepts in the ordered domain is an important issue because it helps to obtain some properties induced from the interaction between topology and algebra. Also, it helps to describe and solve some practical problems; see [8]

To this end, we [16] have formulated the concept of soft topological ordered spaces as an extension of the concept of soft topological spaces. Then we [17] have utilized monotone soft sets to define some soft ordered maps and investigated their main properties. In this work, we have used soft semi open sets to give the concepts of soft $x$-semi continuous, soft $x$-semi open, soft $x$-semi closed and soft $x$-semi homeomorphism maps for $x \in\{I, D, B\}$. We have given various characterizations for these concepts and have shown the relationships among them with the help of examples. It should be noted that results obtained herein and results obtained in [14] are independent of each other. Also, they are special case of results obtained in $[32,19]$ and are genuine generalizations of results obtained in [18]. 


\section{References}

[1] M. Abo-Elhamayel and T. M. Al-shami, Supra homeomorphism in supra topological ordered spaces, Facta Universitatis, Series: Mathematics and Informatics, 31 (5) (2016), 1091-1106.

[2] S. Al-Ghour, Soft $\omega^{*}$-paracompactness in soft topological spaces, International Journal of Fuzzy Logic and Intelligent Systems, 21(1) (2021), $57-65$.

[3] S. Al-Ghour and W. Hamed, On two classes of soft sets in soft topological spaces, Symmetry, 12 (2020), 265; doi:10.3390/sym12020265

[4] T. M. Al-shami, Supra $\beta$-bicontinuous maps via topological ordered spaces, Mathematical Sciences Letters, 6(3) (2017), 239-247.

[5] T. M. Al-shami, Corrigendum to "On soft topological space via semi-open and semi-closed soft sets, Kyungpook Mathematical Journal, 54 (2014) 221-236", Kyungpook Mathematical Journal, 58(3) (2018), 583-588.

[6] T. M. Al-shami, On some maps in supra topological ordered spaces, Journal of New Theory, 20 (2018), 76-92.

[7] T. M. Al-shami, Soft somewhere dense sets on soft topological spaces, Communications of the Korean Mathematical Society, 33(4) (2018), 13411356.

[8] T. M. Al-shami, Compactness on soft topological ordered spaces and its application on the information system, Journal of Mathematics, Volume 2021, Article ID 6699092, 12 pages.

[9] T. M. Al-shami, Sum of the spaces on ordered setting, Moroccan Journal of Pure and Applied Analysis, 6(2) (2020), 255-265.

[10] T. M. Al-shami and M. Abo-Elhamayel, Novel class of ordered separation axioms using limit points, Applied Mathematics \& Information Sciences, 14(6)(2020), 1103-1111.

[11] T. M. Al-shami, I. Alshammari and B. A. Asaad, Soft maps via soft somewhere dense sets, Filomat, 34(10)(2020), 3429-3440.

[12] T. M. Al-shami, M. A. Al Shumrani and C. Özel, Another form of supra ordered separation axioms, Journal of Mathematical Extension, 15(1) (2021), 105-125. 
[13] T. M. Al-shami and M. E. El-Shafei, On supra soft topological ordered spaces, Arab Journal of Basic and Applied Sciences, 26(1) (2019), 433 445.

[14] T. M. Al-shami and M. E. El-Shafei, Some types of soft ordered maps via soft pre open sets, Applied Mathematics \& Information Sciences, 13(5) (2019), 707-715.

[15] T. M. Al-shami, M. E. El-Shafei and M. Abo-Elhamayel, Almost soft compact and approximately soft Lindelöf spaces, Journal of Taibah University for Science, 12(5) (2018), 620-630.

[16] T. M. Al-shami, M. E. El-Shafei and M. Abo-Elhamayel, On soft topological ordered spaces, Journal of King Saud University-Science, 31(4) (2019), 556-566.

[17] T. M. Al-shami, M. E. El-Shafei and M. Abo-Elhamayel, On soft ordered maps, General letters in Mathematics, 5(3) (2018), 118-131.

[18] T. M. Al-shami, M. E. El-Shafei and M. Abo-Elhamayel, New types of soft ordered mappings via soft $\alpha$-open sets, Italian Journal of Pure and Applied Mathematics, 42 (2019), 357-375.

[19] T. M. Al-shami, M. E. El-Shafei and B. A. Asaad, Other kinds of soft $\beta$ mappings via soft topological ordered spaces, European Journal of Pure and Applied Mathematics, 12(1) (2019), 176-193.

[20] T. M. Al-shami and L. D. R. Kočinac, The equivalence between the enriched and extended soft topologies, Appl. Comput. Math., 18(2) (2019), 149-162.

[21] M. I. Ali, F. Feng, X. Liu, W. K. Min and M. Shabir, On some new operations in soft set theory, Computers and Mathematics with Applications, 57 (2009), 1547-1553.

[22] A. Aygünoğlu and H. Aygün, Some notes on soft topological spaces, Neural Computers and Applications, 21 (2012), 113-119.

[23] B. A. Asaad, M. K. Tahat, T. M. Al-shami, Supra b maps via topological ordered spaces, European Journal of Pure and Applied Mathematics, 12 (3) (2019), 1231-1247. 
[24] B. Chen, Soft semi-open sets and related properties in soft topological spaces, Applied Mathematics \& Information Sciences, 7(1) (2013), 287294.

[25] B. Chen, Some local properties of soft semi-open sets, Discrete Dynamics in Nature and Society, Volume 2013, Article ID 298032, 6 pages.

[26] P. Das, Separation axioms in ordered spaces, Soochow Journal of Mathematics, 30(4) (2004), 447-454.

[27] S. Das and S. K. Samanta, Soft metric, Annals of Fuzzy Mathematics and Informatics, 6(1) (2013), 77-94.

[28] M. E. El-Shafei, M. Abo-Elhamayel and T. M. Al-shami, Strong separation axioms in supra topological ordered spaces, Mathematical Sciences Letters, 6(3) (2017), 271-277.

[29] M. E. El-Shafei, M. Abo-Elhamayel and T. M. Al-shami, Partial soft separation axioms and soft compact spaces, Filomat, 32(13) (2018), 47554771.

[30] M. E. El-Shafei and T. M. Al-shami, Applications of partial belong and total non-belong relations on soft separation axioms and decision-making problem, Computational and Applied Mathematics, 39(3) (2020),138, https://doi.org/10.1007/s40314-020-01161-3

[31] M. E. El-Shafei and T. M. Al-shami, Some operators of a soft set and soft connected spaces using soft somewhere dense sets, Journal of Interdisciplinary Mathematics, (2021), https://doi.org/10.1080/ 09720502.2020.1842348

[32] M. E. El-Shafei and T. M. Al-shami, Some new types of soft b-ordered mappings, International Journal of Advances in Mathematics, Volume 2019 (3) (2019), 1-14.

[33] A. Kandil, O. A. E. Tantawy, S. A. El-Sheikh and A. M. Abd El-latif, $\gamma$-operation and decompositions of some forms of soft continuity in soft topological spaces, Annals of Fuzzy Mathematics and Informatics, 7(2) (2014), 181-196.

[34] A. Kandil, O. A. E. Tantawy, S. A. El-Sheikh and A. M. Abd El-latif, Soft semi separation axioms and some types of soft functions, Annals of Fuzzy Mathematics and Informatics, 8(2) (2014), 305-318. 
[35] M. K. R. S. V. Kumar, Homeomorphism in topological ordered spaces, Acta Ciencia Indica, XXVIII(M)(1)(2012), 67-76.

[36] F. Lin, Soft connected spaces and soft paracompact spaces, International Journal of Mathematical Science and Engineering, 7(2) (2013), 1-7.

[37] J. Mahanta and P. K. Das, On soft topological space via semi-open and semi-closed soft sets, Kyungpook Mathematical Journal, 54 (2014), 221236.

[38] P. K. Maji, R. Biswas and R. Roy, Soft set theory, Computers and Mathematics with Applications, 45 (2003), 555-562.

[39] S. D. McCartan, Separation axioms for topological ordered spaces, Mathematical Proceedings of the Cambridge Philosophical Society, 64 (1986), 965-973.

[40] D. Molodtsov, Soft set theory-first results, Computers and Mathematics with Applications, 37 (1999), 19-31.

[41] L. Nachbin, Topology and order, D. Van Nostrand Inc. Princeton, New Jersey, (1965).

[42] S. Nazmul and S. K. Samanta, Neigbourhood properties of soft topological spaces, Annals of Fuzzy Mathematics and Informatics, 6(1) (2013), 1-15.

[43] K. K. Rao and R. Chudamani, Semi-homeomorphism in topological ordered spaces, International Journal of Mathematical Sciences, Technology and Humanities, 39 (2012), 383-397.

[44] W. Rong, The countabilities of soft topological spaces, International Journal of Mathematical, Computational, Physical, Electrical and Computer Engineering, 6(8) (2012), 952-955.

[45] M. Shabir and M. Naz, On soft topological spaces, Computers and Mathematics with Applications, 61 (2011), 1786-1799.

[46] I. Zorlutuna, M. Akdag, W. K. Min and S. K. Samanta, Remarks on soft topological spaces, Annals of Fuzzy Mathematics and Informatics, 2 (2012), 171-185. 